\title{
Reconsidering Social Dynamics: Fundamentality and Social Simulations
}

\author{
loannis Katerelos* and Charalambos Tsekeris**
}

\author{
Department of Psychology, Panteion University of Social and Political Sciences, Athens, Greece \\ *iokat@panteion.gr, **tsekeris@gmail.com
}

Abstract: We live in a ceaselessly changing and inescapably dynamic social world. Given the inherent unpredictability of human complex systems, this brief article seeks to show that agent-based social simulations can possibly approach the ideal of a fundamental law of social dynamics, including all forms or processes of social dynamics, articulated with everyday life and action, individual or collective. This ultimately tends to recover the explanatory potential of social networks and offer an efficient research basis for the creative re-conceptualization of social dynamics.

Keywords: Human Complex Systems, Chaos Theory, Social Dynamics, Fundamental Laws, Unpredictability, Social Simulations, Agent-Based Modelling, Emergence, Social Networks, Social Research Methods, Social Behaviour

Acknowledgement: This reflection presents personal views of the authors. It is a modified version of a paper presented at the First Panhellenic Conference of the Hellenic Sociological Society (HSS), which was held January 16-17, 2009 in Athens, Greece.

Indoctrinating fundamentality within the wide field of the social sciences seems to be a very difficult and risky endeavor. Increasingly, social scientists tend to promiscuously formulate relativistic claims in terms of a ubiquitous "social context" or of a "discursive approach" to social facts, or of the inherently "socially constructed" character of reality. Yet, is there any space for a (transcontextual) fundamental law of social dynamics?

In principle, a fundamental law of social dynamics should generally encompass all forms or processes of social dynamics and should arguably be articulated with everyday human life, individual or collective, regardless of culture, religion, values, opinions or beliefs. Nevertheless, this must not involve any naïve normativism, or uncritical implications of social engineering, since free will, choice and purposeful action seem indispensable (Turner 1997).

It is then implied that any analytical attempt to impose grand deterministic projects upon social action is condemned to inexorably fail due to the "human nature" itself. Nobody can actually, voluntaristically or not, situate her/himself above social/societal (relational) dynamics, independent of her/his "strong will and conviction", intelligence or charisma, methodological approach or research skills, prestige, authority or political power, social status or symbolic capital, institutional position (centrality) or epistemological standpoint. ${ }^{1}$

As Stacey, Griffin and Shaw comprehensively argue and conclude, "...no individual or group of individuals can be 'in control' of the whole system. This departs from the dominant discourse in which the only alternative to an individual being 'in control' is thought to be anarchy" (Stacey, Griffin, and Shaw 2000, 124). The hitherto obsolete deterministic platform of cause and effect, leading to predictions, seems to be incompatible to the above theoretical framework. ${ }^{2}$ Interestingly, it is the predictability discourse itself that excludes free will and choice, because it sees social facts as by nature or de facto inescapable and any purposeful action as almost meaningless.

In the early 1960s, a new analytical approach began to gain ground which, without denying the basic tenets of determinism, demonstrated that not all deterministic systems are predictable, because some of them are sensitive to initial conditions. This means that small, or even trivial, causes

1 See the very interesting critical discussion of what Dick Pels perceptively calls "methodological voluntarism" (Pels 2000, 210-214).

${ }^{2}$ A quite simple mathematical analysis could easily show that, even in simple and explainable systems, which perfectly obey Newton's laws of motion, we cannot always and accurately predict what is going to happen next. This is because of a persistent instability and fluidity, as well as of an undecidable multiplicity of forces that variously affect and act upon an object. For sure, any attempt to predict a simple system's future behaviour over long times will be defeated. Of course, this does not mean that we can say nothing about the very dynamic properties and processes of the system. Although the scientific "ability to predict unpredictability" would be a very useful tool in policy making (Saperstein 1986), unpredictability itself cannot be predicted - at least not in a traditional sense, that is, by comparing successive snapshots of a system's trajectory in time (Katerelos and Koulouris 2004a). 
can have disproportional (unanticipated and unforeseen) effects upon the behavior of the entire system. Such an approach was named by E. Lorenz as chaos theory (Lorenz 1963) and was widely communicated to the public by J. Gleick's best-seller Chaos: Making a New Science, nearly two decades later (Gleick 1987). ${ }^{3}$

A chaotic system's esoteric interactions usually prevail upon external control attempts. The counter-intuitive behaviours of human complex systems generally result from often "very complicated feedback loops in the system, which cause many management mistakes and undesired side effects. Such effects are particularly well-known from failing political attempts to improve the social or economic conditions" (Helbing 2009, 428).

As John Urry rightly observes, many small "local actions" can rapidly interact and surprisingly ramify to create "global waves" or "global fluids" (i.e. unstable networks such as travelling peoples, automobility, global brands, social and political movements, environmental and health hazards), which are seen as highly unpredictable, and as often lacking a clear starting and end point: "The 'particles' of people, information, objects, money, images, risks and networks move within and across diverse regions forming heterogeneous, uneven, unpredictable and often unplanned waves...Such waves demonstrate no clear point of departure, deterritorialised movement, at certain speeds and at different levels of viscosity with no necessary end state or purpose" (Urry 2003, $60)$.

Paradoxically, chaos theory had a very limited impact on the social sciences. This was mainly due to its immanent reference to the physical world, as well as to its strong attachment to a rigid positivistic framework. Such an attachment triggered a sharp and decisive division between the interdisciplinary hybrid science of chaos and the realm of social science (or social research). The latter has totally rejected the mechanistic, naturalistic or organicistic connotations of "chaos" and its theoretical and methodological applications to culture and society (Kiel and Elliot 1997).

Since the 1970s, chaos theory has been variously introduced and developed within the wider field of humanities to serve the discourses of post-modernism, post-structuralism, social constructivism, aesthetics, and so on. Nevertheless, this development, mostly articulated in an insular "fuzzy" fashion, has been followed by inappropriate idioms, the diminishment or disappearance of the qualitative or material dimension, and the eventual misunderstanding of what chaos theory really means (Smith and Jenks 2006).

Even after the 1990s, when computing technology was widely diffused and computational social models were rendered highly accessible to most scholars, social research (albeit computer-aided) continued to orient itself to a constructionist mode. Social reality was then "moved to the suburbs" once again - and this time definitely. Hence, fundamentality in the social sciences has been largely abandoned and often perceived as chimerical, given the cultural and value diversity evidenced in contemporary societies throughout the world.

In general, the advent of social simulations, as a new methodological perspective, initially attempted to re-establish the famous connection between micro and macro elements, or between lived social reality and social facts, as pursued by Emile Durkheim in the late nineteenth century (Durkheim 1951). Axelrod comprehensively describes social simulations as a wholly new approach for social sciences and, beyond that, as a third way of doing science (Axelrod 1997). Radically different from induction or deduction, social simulations have the high ambition of presenting a new kind of thinking and, consecutively, new and surprising findings (Friedkin 1998; Epstein and Axtell 1996).

This novel kind, of course, conveys strong positivistic features - namely, objectivism (subject/object distinction), value freedom (facts/values distinction), instrumentalism, and technicism (Gilbert and Doran 1993; Gilbert and Troitzsch 2005). But it also bears a negative positivistic weight - that is, major disadvantages mostly pertaining to the same context. The latter involves a lack of empirical data or, more broadly, a total lack of experiential basis, either in a phenomenalistic sense or in a mere physicalistic way.

This entails that social simulations (as social research methodology) are tightly linked to positivism but, at the same time, a strong abstraction (a "modelization") is necessarily needed to make a direct reference to another social world: a world seen as full of intentions, sentiments, motives,

\footnotetext{
${ }^{3}$ Lorenz's experiments eventually showed that the behaviour of any unstable system (that is, a system that does not exhibit regular or periodic fluctuations) cannot be predicted. Furthermore, his complex graphic representations of this behaviour demonstrated "a kind of infinite complexity [that] stayed within certain bounds, never running off the page but never repeating itself" (Gleick 1987, 30). In the mid-1980s, the Santa Fe Institute (www.santafe.edu) was established to further organize and develop systematic inquiry into complex adaptive systems (CAS).
} 
reasons, constructions and subjectivities ${ }^{4}$ - according to the rationale of hermeneutics, phenomenology, cultural studies, etc.

In such an analytical viewpoint, the social simulation researcher is representing her/his socially shared conception of the social world by modelizing and simulating it (Tsekeris 2009). ${ }^{5}$ Thus, the researcher is subject to certain social variables, factors and influences, or even infected by a "fluid" and "inter-subjective" social reality consisting of the messy contents of human interaction, including rationalizations, values, norms, ethical principles and moral judgments.

What is therefore needed here is to employ a social simulation model based on simplicity and emergence. That is, fundamentality can solely be accessed in a bottom-up way and complexity must result as a research finding. This comes in direct contrast to a top-down procedure, which would inevitably pre-suppose emerging complexity as a starting point, finally leading to a circular argument - a reflexive duplication, or a vicious analytical circle (Pels 2000).

Existing simulation models do not seem to actually correspond to the above analytical framework; either they go from "simple" rules to an emerging yet equally "simple" end, mostly yielding social equilibrium (simplicity in, simplicity out) (Gilbert 2008), or they move from "complex" rules (introducing complexity) to an emerging "complex" outcome, in a rather anticipated way (complexity in, complexity out) (e.g. Flache and Torenvlied 2004).

This kind of dualism is counter-productive and persistent in all social simulation modeling, perhaps with the exception of an agent-based simulation model called MER model (Katerelos and Koulouris 2004a; 2004b). According to the MER model, all social agents have to comply with two simple rules: (a) each agent seeks to reach social consensus (by adopting the mean of his/her surrounding opinions) and (b) each agent attempts to attain internal consistency corresponding to a stabilized internal cognitive state (by maintaining the opinion which sustained the major social change and abstracting this change from the counter-opinion). ${ }^{6}$

A system in complete (or absolute) equilibrium cannot possibly give any information about its internal functions or mechanisms: if there are absolutely no changes occurring, then nothing would serve as a trigger to study the regularities that govern it. So, systemic equilibrium almost amounts to complete methodological blindness. It now makes sense that a "stable system" is neither a feasible nor a desirable condition, since a predictable, motionless and inert social system does not exhibit any characteristics of adaptation, creativity or innovation; it is therefore doomed to die (or dissolute). This would probably be "a very hopeless, colourless, periodical, monotonous, dull and boring world: A completely grey social universe (against human nature itself)" (Tsekeris 2010, 39).

Yet, high instability (or high entropy) is an equally undesirable condition, since the system cannot adequately evaluate or integrate, or even digest, the continuous flow of change and innovation. The reason is that a very unstable social system does not have the necessary time of transforming innovation to adaptation. Such a system is rather condemned to explode because localities will sooner or later tend to autonomize - perhaps, cutting ties purposefully. This possibly leads to a structural segmentation aimed at local stabilization, since an overall stabilization is completely impossible.

Hence, contrary to the traditional or received criticism against social network theoretical approach as "mere descriptive" (Borgatti, Mehra, Brass, and Labianca 2009), agent-based simulation modeling can fruitfully use social networks as a causal factor shaping social dynamics. In other words, social networks may indeed involve causal inference and not "just methodology". Last but not least, social theorists and researchers ought to maintain a critical explanatory view on the ongoing dialectic of bottom-up and top-down emergence in contemporary complex social systems (Fuchs and Hofkirchner 2005).

\footnotetext{
${ }^{4}$ In a way, social simulations methodology embraces a realist perspective (e.g. Little 1998; Sawyer 2007) but, at the same time, rejects absolute empiricism: the underlying mechanisms (under investigation) do exist; they are considered to be real but they are not necessarily directly observable in real situations. Thus, a direct observation (even when systematic or "methodologically correct") of this world may possibly lead to faulty conclusions due to negligence of the principle of emergence - that is, micro-motives reflexively lead to macro-behaviours (Schelling 1978) - or due to an excessive analytical focus on a particular substantive aspect (for example, discourse and communication), which may be a mere epiphenomenon to the social phenomenon under scrutiny.

${ }^{5}$ Frequently, social researchers are answerable to the so-called "confirmation bias" (Greenwald 2012). This is a rather unchartered analytical territory concerning the social researchers using simulations as their primary approach, although it is a well-analyzed phenomenon in traditional (empirical) methodological approaches.

${ }^{6}$ Thus, an agent permanently oscillates between a "social" equilibrium and an "internal-psychological" equilibrium. In some sense, each time agents "attain" social equilibrium (consensus), the psychological equilibrium mechanism destabilizes and differentiates them into one of the previously socially stable dimensions. Of course, being desynchronized exhibits the need for social coordination once again. This is a perpetual systemic motion which can show different types of dynamics.
} 


\section{References}

Axelrod, Robert. 1997. Advancing the Art of Simulation in the Social Sciences. Complexity 3 (2): 16-22.

Borgatti, Stephen, Ajay Mehra, Daniel Brass, and Giuseppe Labianca. 2009. Network Analysis in the Social Sciences. Science 323: 892-895.

Durkheim, Emil. 1951. Suicide: A Study in Sociology. New York: Free Press.

Epstein, Joshua, and Robert Axtell. 1996. Growing Artificial Societies. Cambridge: MIT Press.

Flache, Andreas, and Rene Torenvlied. 2004. When Will They Ever Make Up Their Minds? The Social Structure of Unstable Decision Making. Journal of Mathematical Sociology 28: 171-196.

Friedkin, Noah. 1998. A Structural Theory of Social Influence. Cambridge: Cambridge University Press.

Fuchs, Christian, and Wolfgang Hofkirchner. 2005. The Dialectic of Bottom-up and Top-down Emergence in Social Systems. tripleC - Cognition, Communication, Co-operation: Open Access Journal for a Global Sustainable Information Society 3 (2): 28-50. Accessed April 3, 2012. http://www.triple-c.at/index.php/tripleC/article/view/21/20

Gilbert, Nigel. 2008. Agent Based Models. Los Angeles: Sage.

Gilbert, Nigel, and Jim Doran. 1993. Simulating Societies: The Computer Simulation of Social Phenomena. London: UCL Press.

Gilbert, Nigel, and Klaus Troitzsch. 2005. Simulation For the Social Scientist. London: Open University Press.

Gleick, James. 1987. Chaos: Making a New Science. New York: Penguin.

Greenwald, Anthony. 2012. There Is Nothing So Theoretical As a Good Method. Perspectives on Psychological Science 7: 99-108.

Helbing, Dirk. 2009. Managing Complexity in Socio-Economic Systems. European Review 17 (2): 423-438.

Katerelos, Ioannis, and Andreas Koulouris. 2004a. Is Prediction Possible? Chaotic Behaviour of Multiple Equilibria Regulation Model in Cellular Automata Topology. Complexity 10 (1): 23-36.

Katerelos, loannis, and Andreas Koulouris. 2004b. Seeking Equilibrium Leads to Chaos: Multiple Equilibria Regulation Model. Journal of Artificial Societies and Social Simulations 7 (2). Accessed April 3, 2012. http://jasss.soc.surrey.ac.uk/7/2/4.html

Kiel, Douglas, and Euel Elliott, eds. 1997. Chaos Theory in the Social Sciences: Foundation and Application. Ann Arbor: University of Michigan Press.

Little, Daniel. 1998. Micro-Foundations, Method and Causation: On the Philosophy of Social Sciences. New Brunswick: Transaction Publishers.

Pels, Dick. 2000. Reflexivity: One Step Up. Theory, Culture \& Society 17 (3): 1-25.

Saperstein, Alvin. 1986. Predictability, Chaos, and the Transition to War. Bull. Peace Proposals 17 (1): 87-93.

Sawyer, Keith. 2007. Social Emergence: Societies as Complex Systems. New York: Cambridge University Press.

Schelling, Thomas. 1978. Micromotives and Macrobehavior. New York: W. W. Norton and Company.

Smith, John, and Chris Jenks. 2006. Qualitative Complexity. New York: Routledge.

Stacey, Ralph, Douglas Griffin, and Patricia Shaw. 2000. Complexity and Management: Fad or Radical Challenge to SystemsTthinking?. London: Routledge.

Tsekeris, Charalambos. 2009. Advances in Understanding Human Complex Systems. Australian Journal of Basic and Applied Sciences 3 (4): 4040-4045.

Tsekeris, Charalambos. 2010. Chaos and Unpredictability in Social Thought: General Considerations and Perspectives. Sociologija. Mintis ir veiksmas 27 (2): 34-47.

Turner, Frederick. 1997. Forward: Chaos and Social Science. In Chaos, Complexity and Sociology, edited by Raymond Eve, Sara Horsfall and Mary Lee, xi-xxv. Thousand Oaks: Sage.

Urry, John. 2003. Global Complexity. Cambridge: Polity Press.

\section{About the Author}

\section{loannis Katerelos}

studied Sciences of Education in the University of Marseille-Aix en Provence, France, where he obtained his Doctoral Degree in Social Psychology. Since 2004, he has been Associate Professor of Social Psychology at the Department of Psychology, Panteion University of Social and Political Sciences, Athens, Greece. His academic interests involve research methods, with particular emphasis on the dynamics of social representations, simulation methodology and Chaos/Complexity Theory in the Social Sciences.

Charalambos Tsekeris

graduated with Distinction from Brunel University (West London, UK) and earned his doctoral degree in Reflexivity from Athens Panteion University (Department of Sociology). He is Member of the Hellenic Sociological Society (HSS) and Senior Researcher at the Lab of Virtual Reality, Internet Research \& E-Learning, Department of Psychology, Panteion University of Social and Political Sciences, Athens, Greece. His research interests involve human complex systems, psychosocial studies, epistemology, and social theory. 